\title{
CHAOTIC QUANTIZED FEEDBACK STABILIZERS: THE SCALAR CASE*
}

FABIO FAGNANI ${ }^{\dagger}$

\author{
Dedicated to Sanjoy Mitter on the occasion of his 70th birthday.
}

\begin{abstract}
In this paper we consider practical stabilization strategies of scalar linear systems by means of quantized feedback maps which use a minimal number of quantization levels. These stabilization schemes are based on the chaotic properties of piecewise affine maps and their performance can be analyzed in terms of the mean time needed to shrink the system from an initial interval into a fixed target interval. We show here that this entrance time grows linearly with respect to the contraction rate defined as the quotient of the length of the initial and target interval respectively. Estimations are obtained using denumerable Markov chains arguments.
\end{abstract}

1. Introduction and problem statement. Control problems where the information flow between the plant and the controller is an important feature to be considered in the design, have become very popular in the last few years. See $[1,2,5,6,7,12,13,14]$ and the reference therein. Indeed, information flow becomes important in situations where a channel with limited information rate has to be used between the plant and the controller or when the controller needs to be simple and is only allowed to process a limited number of information per time. We expect that an information flow constraint will in general degrade the performances of the feedback loop scheme and we also expect in general a trade-off between performances and the amount of information exchange allowed in the loop.

The specific problem we consider in this paper is the stabilization problem for a scalar linear system

$$
x_{t+1}=a x_{t}+u_{t} .
$$

We consider memoryless feedback maps $u_{t}=k\left(x_{t}\right)$ which are quantized, namely $k$ is piecewise constant assuming only a finite number $N$ of values. The number $N$ can be thought, in this context, as a measure of the flow of information exchanged, at every time instant, between the system and the controller. The closed loop map

$$
\Gamma(x)=a x+k(x)
$$

is a piecewise affine map with fixed slope $a$. As a consequence, if $|a|>1, \Gamma$ will always exhibit local instability so that asymptotic stabilization can not be reached by means of these type of feedbacks [5]. We here consider a sort of practical stability.

\footnotetext{
*Accepted for publication on March 18, 2004.

$\dagger$ Dipartimento di Matematica, Politecnico di Torino, C.so Duca degli Abruzzi, 24, 10129 Torino, Italy, E-mail: fabio.fagnani@polito.it
} 
Definition: Given two intervals $J \subseteq I$, we say that $\Gamma: \mathbb{R} \rightarrow \mathbb{R}$ is almost $(I, J$,$) -stable$ if

1. $I$ is almost-invariant: for almost every $x_{0} \in I$ (with respect to the uniform probability on $I), \Gamma\left(x_{0}\right) \in I$.

2. $J$ is almost-invariant: for almost every $x_{0} \in J, \Gamma\left(x_{0}\right) \in J$.

3. for almost every $x_{0} \in I$, there exists an integer $t \geq 0$ such that $\Gamma^{t}\left(x_{0}\right) \in J$. A quantized feedback map $k: \mathbb{R} \rightarrow \mathbb{R}$ is said to be almost $(I, J)$-stabilizing if the corresponding closed loop map $\Gamma$ is $(I, J)$-stable.

The reason for the use of 'almost' is that in previous works $[7,8]$ we have also considered the corresponding stronger definition of $(I, J)$-stability where the three properties are asked to hold for every $x_{0}$. In this paper we will not use this stronger definition, however we have preferred to maintain the old terminology in order not to create confusion with these other works.

In this paper we will restrict to the situation when both $I$ and $J$ are symmetric intervals with respect to the 0 . Notice that linearity yields the following scaling property: if $k(x)$ is $(I, J)$ - stabilizing, then, $\eta k\left(\eta^{-1} x\right)$ is $(\eta I, \eta J)$ - stabilizing. This observation allows to restrict ourselves to study the almost stabilization problem for intervals of type $I=[-1,1]$ and $J=[-\epsilon, \epsilon]$. The ratio $C=1 / \epsilon$ is called the contraction rate of the given stabilization scheme.

By varying among all the possible feedback quantized maps $k$, the closed loop maps $\Gamma$ which we obtain are all the possible piecewise affine maps with all branches having fixed slope $a$ and with only a finite number of discontinuities. From this observation, it is easy too see [5] that, given any interval $I$, we can make it almostinvariant for the closed loop map $\Gamma$ by means of a quantized feedback map $k$ which has $\lceil|a|\rceil$ quantization levels inside $I$ and this is the minimal number of quantization levels needed. Therefore if $\Gamma$ is almost $(I, J)$-stable, there will be always at least $\lceil|a|\rceil$ quantization levels inside $J$. The real parameter of interest thus become in this context the number of quantization intervals inside $I \backslash J$ which, from now on will always be denoted by $N$. In [7] it was shown that $\lceil|a|\rceil$ quantization levels inside $I \backslash J$ are also sufficient, to achieve almost $(I, J)$-stability disregarding how large we have chosen the contraction rate $C$. These stabilization schemes are based on chaotic properties of expansive piecewise affine maps.

The performance of a stabilization scheme in this context can be measured through the entrance time in the target interval $J$. The first entrance time function

$$
T_{J}: I \rightarrow \mathbb{N} \cup\{+\infty\}
$$

is defined by

$$
T_{J}(x)=\inf \left\{t \in \mathbb{N} \mid \Gamma^{t} x \in J\right\}
$$


and by $T_{J}(x)=+\infty$ if $\Gamma^{t} x \notin J$ for all $t$. The map $T_{J}$ is almost surely finite if we have almost $(I, J)$-stability. Finally, we define

$$
T=\mathbb{E}\left(T_{J}\right)
$$

the mean value with respect to the uniform probability density on $I$. For the stabilization schemes proposed in [7] and using $\lceil|a|\rceil$ levels, we have shown that there exists a constant $K>0$, only depending on the slope $a$, such that for the chaotic stabilization schemes previously considered the following bound holds true:

$$
T \leq K C \ln C
$$

Of course, there exist many others stabilization schemes [8] all having better performances in terms of the entrance time. There is a scheme, for instance, where $T$ grows only logarithmically as a function of the contraction rate $C$, and others where $T$ is even constant (dead-beat schemes) with respect to $C$. These higher performances are paid in terms of number of quantization levels $N$ which, instead of being constant as in these chaotic schemes, has to grow respectively, logarithmically or linearly in $C$. In [8] we have established quit precise bounds showing how this trade-off between performance and complexity can not be improved. These bounds (in the case when $|a|>2$ ) actually show a surprising symmetry between $T$ and $N$ and they forecast that as there exist stabilization schemes with $T$ constant (asymptotically equal to 1 for $C \rightarrow+\infty$ ), with a number of quantization levels growing linearly with respect to $C$, in the same way there should exist stabilization schemes with a constant number of quantization levels and entrance time $T$ growing linearly in $C$. This seems to indicate that (4) is not tight or, possibly, that there should be stabilization schemes with the same number of quantization levels but with better time performance. In the case when $a \in \mathbb{Z}$ and $|a| \geq 2$, we have explicitely constructed such stabilization schemes in [8]. The main goal of this paper is to extend this construction (with just the addition of one quantization level) to the case of any $a$ with the condition $|a|>2$. The following is the main result of this paper.

Theorem 1. Let $a$ be such that $|a|>2$. Let $I=[-1,1]$ and $J=[-\epsilon, \epsilon]$ where $0<\epsilon<1$. Then, there exists a quantized almost $(I, J)$-stabilizing feedback map $k: I \rightarrow \mathbb{R}$ with a number of quantization levels inside $I \backslash J, N \leq\lceil|a|\rceil+1$. Moreover, we have the following estimation for the mean entrance time

$$
T \leq K C,
$$

where $K$ is a positive constant only depending on a (and not on $\epsilon$ ) and where $C=1 / \epsilon$ is the contraction rate.

In the rest of this section we introduce the class of quantized feedback maps we will consider throughout the paper and we outline the content of the following sections. 
1.1. Chaotic quantized feedback strategies. Let $k_{I}$ be a quantized feedback making the interval $I$ almost invariant: there are many possibilities and, as already mentioned, the minimal number of quantization levels needed is $\lceil|a|\rceil$. Let $\Gamma_{I}$ be the corresponding closed loop map. Let $J \subseteq I$ be another interval which is not in general invariant by $\Gamma_{I}$. Let $k_{J}$ be another quantized feedback making the interval $J$ almost invariant and let $\Gamma_{J}$ be the corresponding closed loop map. Consider now the new quantized feedback $k_{(I, J)}$ obtained by amalgamating the previous two, formally defined by

$$
k_{(I, J)}(x)= \begin{cases}k_{J}(x) & x \in J \\ k_{I}(x) & x \in I \backslash J\end{cases}
$$

and let $\Gamma_{(I, J)}$ be the corresponding closed loop map. Clearly, $\Gamma_{(I, J)}$ leaves both $I$ and $J$ almost invariant. It is clear that $\Gamma_{(I, J)}$ is almost $(I, J)$-stable if and only if the map $\Gamma_{I}$ possesses the following property $(\mathrm{P})$ : for almost every $x \in I$ there exists an integer $t \geq 0$ such that $\Gamma_{I}^{t}(x) \in J$. Notice that this property $(\mathrm{P})$ only concerns the map $\Gamma_{I}$ and the subinterval $J$. It is not difficult to construct a feedback $k_{I}$ such that the corresponding map $\Gamma_{I}$ possess property $(\mathrm{P})$ whatever is $J$ : in [7] there are explicit constructions of such feedbacks requiring just $\lceil|a|\rceil$ quantization levels. The construction proposed here uses, in general, one level more, but it has a symmetric structure which turns out to be very useful in the sequel. Consider the piecewise affine map

$$
\Gamma(x)=a x, \quad x \in\left(-|a|^{-1},|a|^{-1}\right)
$$

extended to $\mathbb{R}$ by $2|a|^{-1}$-periodicity. Notice that $\Gamma(\mathbb{R}) \subseteq[-1,1]$ so that in particular $[-1,1]$ is almost invariant and on it there are only a finite number of discontinuities. For further use we notice that the continuity partition inside $[-1,1]$ consists of the following subintervals:

$$
\begin{aligned}
& I_{k}=\left(-|a|^{-1},|a|^{-1}\right)+2|a|^{-1} k, \quad|k| \leq k_{o}=\left\lfloor\frac{|a|-1}{2}\right\rfloor \\
& I_{k_{o}+1}=\left(|a|^{-1}\left(1+2 k_{o}\right), 1\right) \\
& I_{-k_{o}-1}=\left(-1,-|a|^{-1}\left(1+2 k_{o}\right)\right) .
\end{aligned}
$$

Notice that $I_{k_{o}+1}$ and $I_{-k_{o}-1}$ do not exist if we are in the situation when $a$ is an odd integer. It can be shown that this map possesses property $(\mathrm{P})$ for any subinterval $J \subseteq[-1,1]$ : this will be a consequence of the considerations done in Section 4 .

Notice that the first entrance time map inside $J$ for $\Gamma_{(I, J)}$, coincides with the first entrance time map inside $J$ for $\Gamma_{I}$ (still denoted $T_{J}$ as the first one). In particular, they must have the same mean value $T=\mathbb{E}\left(T_{J}\right)$. Differently from [7] where we used the decay of correlation results in [11], in this paper we will use symbolic dynamics and Markov chains to estimate $T$. In Section 2 we will introduce the symbolic dynamics formalism and the denumerable Markov chain associated with the piecewise affine 
maps. This will allow to reformulate the problem in terms of the entrance time for Markov chains. Section 3 is devoted to a detailed analysis of the spectral properties of the Markov chain associated to our piecewise affine map. Finally, Section 4 contains the specific entrance time estimations and the application to the proof of Theorem 1.

2. Symbolic dynamics and Markov chains. We first make some comments on our definition of quantized map. The feedback map $k: \mathbb{R} \rightarrow \mathbb{R}$ is piecewise constant, more precisely, there exist open disjoint intervals $I_{1}, I_{2}, \ldots, I_{N}$ whose union is dense in $\mathbb{R}$ and elements $u_{1}, u_{2}, \ldots, u_{N} \in \mathbb{R}$ such that

$$
k(x)=u_{j} \quad \text { if } x \in I_{j} .
$$

We do not define $k$ at the boundary points of the quantization intervals. As a consequence the corresponding closed loop map $\Gamma$ is also not defined at the boundary points. To be able to consider powers of $\Gamma$ we have to restrict ourselves to the subset

$$
\Omega=\mathbb{R} \backslash \bigcup_{j=1}^{N} \bigcup_{k=0}^{\infty} \Gamma^{-k} \partial I_{j}
$$

which differs from $\mathbb{R}$ for an utmost denumerable set of points. It is clear that for the type of properties we want to study, almost stability, mean entrance time, it is completely satisfactory to work with $\Omega$ and in the sequel we will always assume that $\Gamma$ is evaluated at points in $\Omega$. If the interval $I$ is almost-invariant for the map $\Gamma$, we will write, with little abuse of notation, $\Gamma: I \rightarrow I$. Moreover, if we are only interested in the behavior of $\Gamma$ inside $I$, we will disregard how it is defined outside, and with $N$ we will denote the number of quantization intervals inside $I$.

The dynamical and ergodic properties of piecewise affine maps as $\Gamma$ introduced in (2) have been extensively studied in the past $[10,4,11,3]$.

Consider a piecewise affine map $\Gamma: I \rightarrow I$ (namely $I$ is assumed to be almostinvariant). Let $\mathcal{I}_{0}=\left\{I_{1}, I_{2}, \ldots, I_{N}\right\}$ be the partition of the interval $I$ into the maximal open subintervals on which $\Gamma$ is affine (this partition is called the minimal partition). In the sequel we will need to consider different partitions of $I$. A partition $\mathcal{I}$ of $I$ into open subintervals is called $\Gamma$-compatible if for every $A \in \mathcal{I}$ we have that $\Gamma$ is affine on $A$. Clearly, $\mathcal{I}_{o}$ is $\Gamma$-compatible and all other $\Gamma$-compatible partitions are obtained by refining the minimal partition. Starting from a given $\Gamma$-compatible partition $\mathcal{I}$, we can construct other $\Gamma$-compatible partitions by defining the so called powers of $\mathcal{I}$ as

$$
\mathcal{I}^{t}=\left\{I_{j_{0}} \cap \Gamma^{-1}\left(I_{j_{1}}\right) \cap \cdots \cap \Gamma^{-t}\left(I_{j_{t}}\right), j_{0}, j_{1}, \ldots, j_{t} \in\{1,2, \ldots, N\}\right\} .
$$

Particularly important will be the powers of the minimal partition $\mathcal{I}_{o}^{t}$.

Fix a partition $\mathcal{I}$. With the pair $(\Gamma, \mathcal{I})$, we can associate the language $\Sigma^{*}(\Gamma, \mathcal{I})$ which is the subset of $\mathcal{I}^{*}$ (the set of all finite words over the finite alphabet $\mathcal{I}$ ) consisting of all the finite words $\omega_{0} \omega_{1} \cdots \omega_{n} \in \mathcal{I}^{*}$ such that

$$
\omega_{0} \cap \Gamma^{-1} \omega_{1} \cap \cdots \cap \Gamma^{-n} \omega_{n} \neq \emptyset .
$$


Consider now the directed graph with set of vertices $\Sigma^{*}(\Gamma, \mathcal{I})$ and set of edges $\mathcal{E}$ given by

$$
\left(\omega_{0} \omega_{1} \cdots \omega_{n} \rightarrow \omega_{0} \omega_{1} \cdots \omega_{n} \omega_{n+1}\right) \in \mathcal{E} \quad \Longleftrightarrow \quad \omega_{0} \omega_{1} \cdots \omega_{n} \omega_{n+1} \in \Sigma^{*}(\Gamma, \mathcal{I}) .
$$

Consider moreover the following labelling $\xi$ on the edges

$$
\xi\left(\omega_{0} \omega_{1} \cdots \omega_{n} \rightarrow \omega_{0} \omega_{1} \cdots \omega_{n} \omega_{n+1}\right)=\omega_{n+1} .
$$

Notice that $\Sigma^{*}(\Gamma, \mathcal{I})$ coincides with the set of all the labelled sequences associated with the finite paths on the graph starting from the empty word $\epsilon$. We have thus obtained a Markov representation of our subshift. This representation can be simplified by introducing an equivalence relation on the vertices. With each finite word $\omega_{0} \omega_{1} \cdots \omega_{n} \in \Sigma^{*}(\Gamma, \mathcal{I})$, we associate its symbolic future

$$
\operatorname{fut}_{\Sigma}\left(\omega_{0} \omega_{1} \cdots \omega_{n}\right)=\left\{\bar{\omega}_{0} \bar{\omega}_{1} \cdots \bar{\omega}_{k} \mid \bar{\omega}_{0}=\omega_{n} \text { and } \omega_{0} \omega_{1} \cdots \omega_{n} \bar{\omega}_{1} \cdots \bar{\omega}_{k} \in \Sigma^{*}(\Gamma, \mathcal{I})\right\}
$$

which is a subset of $\Sigma^{*}(\Gamma, \mathcal{I})$. More roughly, the symbolic future of a word $\omega_{0} \omega_{1} \cdots \omega_{n}$ is the set of words whose concatenation with $\omega_{0} \omega_{1} \cdots \omega_{n}$ is in the language $\Sigma^{*}(\Gamma, \mathcal{I})$.

Consider also the geometric future which is

$$
\operatorname{fut}\left(\omega_{0} \omega_{1} \cdots \omega_{n}\right)=\Gamma^{n}\left(\omega_{0} \cap \Gamma^{-1} \omega_{1} \cap \cdots \cap \Gamma^{-n} \omega_{n}\right) .
$$

The following result is in [3], see [8] for a detailed proof.

Proposition 1. Let $\omega_{0} \omega_{1} \cdots \omega_{n}$ and $\nu_{0} \nu_{1} \cdots \nu_{m}$ be two words in $\Sigma^{*}(\Gamma, \mathcal{I})$. Then

$$
\begin{gathered}
\operatorname{fut}\left(\omega_{0} \omega_{1} \cdots \omega_{n}\right)=\operatorname{fut}\left(\nu_{0} \nu_{1} \cdots \nu_{m}\right) \\
\Longleftrightarrow \operatorname{fut}_{\Sigma}\left(\omega_{0} \omega_{1} \cdots \omega_{n}\right)=\operatorname{fut}_{\Sigma}\left(\nu_{0} \nu_{1} \cdots \nu_{m}\right) .
\end{gathered}
$$

Now define $\mathbf{X}_{\mathcal{I}}$ to be the quotient of the set $\Sigma^{*}(\Gamma, \mathcal{I})$ by the equivalence relation

$$
\omega_{0}^{\prime} \cdots \omega_{n}^{\prime} \equiv \omega_{0}^{\prime \prime} \cdots \omega_{m}^{\prime \prime} \Leftrightarrow \operatorname{fut}_{\Sigma}\left(\omega_{0}^{\prime} \cdots \omega_{n}^{\prime}\right)=\operatorname{fut}_{\Sigma}\left(\omega_{0}^{\prime} \cdots \omega_{m}^{\prime}\right)
$$

The elements of $\mathbf{X}_{\mathcal{I}}$ will be called states. The symbol $\left\langle\omega_{0} \omega_{1} \cdots \omega_{n}\right\rangle$ represent the state coinciding with the equivalent class associated with the word $\omega_{0} \omega_{1} \cdots \omega_{n} \in \Sigma^{*}(\Gamma, \mathcal{I})$. The equivalence relation defining $\mathbf{X}_{\mathcal{I}}$ ensures that any state $\mathbf{x}$ has a well defined geometric future fut $(\mathbf{x})$.

Edges and labels can be naturally redefined on $\mathbf{X}_{\mathcal{I}}$ to obtain a new labelled graph which is still a Markov representation of $\Sigma^{*}(\Gamma, \mathcal{I})$ and so with the property that the labelled sequences associated to the finite paths on this graph, starting from empty word, correspond to all the possible sequences in $\Sigma^{*}(\Gamma, \mathcal{I})$. Elements in $\mathbf{X}_{\mathcal{I}}$ will be denoted, from now on, with bold faces letters like $\mathbf{x}$ or $\mathbf{y}$.

We now introduce few notation which will used later on.

$$
\mathbf{x} \in \mathbf{X}_{\mathcal{I}}, \quad \nu(\mathbf{x})=\min \left\{n \in \mathbb{N} \mid \exists \omega_{0} \omega_{1} \cdots \omega_{n} \in \Sigma^{*}(\Gamma, \mathcal{I}) \text { with } \mathbf{x}=<\omega_{0} \omega_{1} \cdots \omega_{n}>\right\}
$$




$$
\mathbf{X}_{\mathcal{I}, P}=\left\{\mathbf{x} \in \mathbf{X}_{\mathcal{I}} \mid \nu(\mathbf{x})=1\right\}=\{\langle\omega\rangle \mid \omega \in \mathcal{I}\} .
$$

$\mathcal{X}_{\mathcal{I}, P}$ is called the set of principal states. It will also be useful to consider the transition matrix associated with the graph over $\mathbf{X}_{\mathcal{I}}$ : if $\mathbf{x}$ and $\mathbf{y}$ are in $\mathbf{X}_{\mathcal{I}}$, we define $t_{\mathbf{x y}}$ equal 1 if there is an edge connecting $\mathbf{x}$ to $\mathbf{y}, 0$ otherwise.

For the sake of simplicity, whenever the partition $\mathcal{I}$ is fixed and clear from the context, we will use the simplified notation $\mathbf{X}$ and $\mathbf{X}_{P}$.

2.1. The canonical Markov chain. Suppose the partition $\mathcal{I}$ has been fixed. We now introduce a transition probability matrix (namely a Markov chain) indexed by the vertices of $\mathbf{X}=\mathbf{X}_{\mathcal{I}}$ by defining $P_{\mathbf{x y}}$ as the probability that $\Gamma(x)$ is in $\operatorname{fut}(\mathbf{y})$ assuming that $x$ is uniformly distributed over fut(x). More formally,

$$
P_{\mathbf{x y}}=\frac{\mathbb{P}\left(\text { fut }(\mathbf{x}) \cap \Gamma^{-1}(\text { fut }(\mathbf{y}))\right.}{\mathbb{P}(\text { fut }(\mathbf{x}))}=t_{\mathbf{x y}} \frac{\mathbb{P}(\text { fut }(\mathbf{y}))}{|a| \mathbb{P}(\text { fut }(\mathbf{x}))},
$$

where $\mathbb{P}$ denotes the uniform probability on $I$. The pair $(\mathbf{X}, P)$ is called the Markov chain associated with $(\Gamma, \mathcal{I})$. With a slight abuse of notation with the symbol $P$ we will also denote the isometric positive operator induced by the Markov chain $P$ :

$$
\begin{gathered}
P: l^{1}(\mathbf{X}) \rightarrow l^{1}(\mathbf{X}) \\
\pi \in l^{1}(\mathbf{X}) \mapsto(P \pi)(\mathbf{y})=\sum_{\mathbf{x} \in \mathbf{X}} \pi(\mathbf{x}) P_{\mathbf{x y}} .
\end{gathered}
$$

Given any probability measure $\pi$ on $\mathbf{X}$, we obtain a stochastic Markov process $X_{n}^{\pi}$ on $\mathbf{X}$ whose finite distributions are

$$
\mathbb{P}_{\pi}\left(X_{0}^{\pi}=\mathbf{x}_{0}, X_{1}^{\pi}=\mathbf{x}_{1}, \cdots, X_{n}^{\pi}=\mathbf{x}_{n}\right)=\pi\left(\mathbf{x}_{0}\right) P_{\mathbf{x}_{0} \mathbf{x}_{1}} P_{\mathbf{x}_{1} \mathbf{x}_{2}} \cdots P_{\mathbf{x}_{n-1} \mathbf{x}_{n}}
$$

$\pi$ is called the initial probability distribution. From now on, we will speak of Markov process when the initial probability distribution has been fixed reserving the term Markov chain for the pair $(\mathbf{X}, P)$.

We now show how the entrance time problem can be reformulated in terms of the Markov chain. We denote by $\pi_{\mathrm{u}} \in l^{1}(\mathbf{X})$ the probability corresponding to the uniform probability on $I$, precisely defined as:

$$
\begin{array}{ll}
\pi_{\mathrm{u}}(<\omega>)=\mathbb{P}(\omega) & \\
\pi_{\mathrm{u}}(\mathbf{x})=0 & \text { if } \nu(\mathbf{x})>1 .
\end{array}
$$

Let now $J=$ fut $(\mathbf{x}) \subseteq I$ be a subinterval, not necessarily invariant by $\Gamma$. Put

$$
\mathbf{X}_{J}=\{\mathbf{y} \in \mathbf{X} \mid \operatorname{fut}(\mathbf{y}) \subseteq J\}
$$

Notice that

$$
\mathbb{P}\left(\Gamma^{n} x \in J\right)=\mathbb{P}_{\pi_{\mathrm{u}}}\left(X_{n}^{\pi_{\mathrm{u}}} \in \mathbf{X}_{J}\right)
$$


Hence, if we denote by $T_{\mathbf{X}_{J}}$ and $T_{\mathbf{x}}$ the first entrance time of the Markov process $X^{\pi_{u}}$ inside, respectively, $\mathbf{X}_{J}$ and $\{\mathbf{x}\}$, we have that

$$
T=\mathbb{E}\left(T_{J}\right)=\mathbb{E}_{\pi_{\mathrm{u}}}\left(T_{\mathbf{X}_{J}}\right) \leq \mathbb{E}_{\pi_{\mathrm{u}}}\left(T_{\mathbf{x}}\right) .
$$

Our goal will be to estimate the last term in (10) in order to obtain an estimation of $T$.

3. The spectral properties of the Markov chain $P$. In order to be able to fruitfully use the discrete symbolic viewpoint we first need to recall a number of concepts from the theory of denumerable chains and to establish a number of specific results for this particular type of Markov chains.

We refer to [9] for the results that we will use. First we recall few definitions. Two states $\mathbf{x}$ and $\mathbf{y}$ are said to be related iff there exist $t_{1}$ and $t_{2}$ such that $P_{\mathbf{x y}}^{t_{1}}>0$ and $P_{\mathbf{y x}}^{t_{2}}>0 . \quad P$ is said to be indecomposable if any pair of states are related to each other. Moreover, given two states $\mathbf{x}$ and $\mathbf{y}$ we denote by $\bar{H}_{\mathbf{x y}}$ the probability of reaching state $\mathbf{y}$ at some time $t \geq 1$, starting from the initial probability distribution $\delta_{\mathbf{x}}$ at $t=0$, and by $N_{\mathbf{x y}}$ the mean number of times we hit state $\mathbf{y}$, starting from the initial probability $\delta_{\mathbf{x}}$, which is the delta probability concentrated on the state $\mathbf{x}$. The following formula holds true

$$
N=\sum_{k=0}^{+\infty} P^{k} .
$$

A state $\mathbf{x} \in \mathbf{X}$ is said to be recurrent iff $\bar{H}_{\mathbf{x} \mathbf{x}}=1$, transient otherwise. In a indecomposable Markov chain there are only two possibilities: either all states are recurrent, or all states are transient. In the first case the Markov chain is said to be recurrent. It can be shown that in the first case $N_{\mathbf{x y}}=+\infty$ for every pair of states, while in the second case $N_{\mathbf{x y}}<+\infty$ for every pair of states. Moreover in the recurrent case we have that $\bar{H}_{\mathbf{x y}}=1$ for every pair of states $\mathbf{x}$ and $\mathbf{y}$. Recurrent states can be of two different types: a state $\mathbf{x}$ is said to be positively recurrent if the mean time of its first return to $\mathbf{x}$ is finite, null recurrent if instead it is infinite. Again, in a recurrent indecomposable chain, either all states are positively or null recurrent. It can be shown that if $P$ is positively recurrent (namely consisting of positively recurrent states), then, there exists exactly one invariant probability measure $\pi_{0}$ for the Markov chain $P$. On the other hand if a Markov chain $P$ admits an invariant probability measure, then this probability is concentrated on the positively recurrent states; in particular, if $P$ is indecomposable, then it must necessarily be positively recurrent.

Finally, $P$ is said to be non-cyclic if it is positively recurrent and, moreover, given any state $\mathbf{x}$, we have that $P_{\mathbf{x x}}^{k}>0$ if $k$ is large enough. If $P$ is non-cyclic, we have convergence to the unique probability measure. Namely, if $\pi$ is any finite measure, we have that

$$
\lim _{n \rightarrow+\infty}\left\|P^{n} \pi-(\pi \cdot e) \pi_{0}\right\|_{1}=0
$$


where $e$ denotes the sequence in $l^{\infty}(\mathbf{X})$ identically equal to 1 and where $($,$) denotes$ the usual duality pairing between $l^{1}$ and $l^{\infty}$.

For finite non-cyclic Markov chains the above convergence is of exponential type and this yields explicit well known formulas to express the mean first entrance time into a target state. These formulas can be generalized to the case of denumerable Markov chains as long as we can recover a type of exponential convergence to the equilibrium probability measure. This of course would hold if we could prove the following structure on the spectrum of the operator $P: 1$ is a simple eigenvalue and the rest of the spectrum is contained inside a ball of radius $r<1$. Unfortunately, in general $P$ will not have such properties at least on the space $l^{1}(\mathbf{X})$. We will need to work on a smaller subset and use the spectral theorem of Ionescu-Tulcea and Marinescu recalled below [4].

Theorem 2. Let $\left(B_{1},\|\cdot\|\right)$ and $\left(B_{2},\||\cdot \||)\right.$ be two Banach spaces with $B_{2} \subseteq B_{1}$ and $B_{2}$ dense in $B_{1}$ (for the norm of $B_{1}$ ). Assume also that the unit ball of $B_{2}$ is contained in the unit ball of $B_{1}$ and it is compact (for the norm of $B_{1}$ ). Let $P: B_{1} \rightarrow B_{1}$ be a linear bounded operator with $\|P\| \leq 1$ and $P\left(B_{2}\right) \subseteq B_{2}$ (and therefore automatically bounded as an operator on $\left.B_{2}\right)$. Assume moreover that there is an integer $q$, a number $\alpha \in[0,1[$ and a number $\beta>0$ such that

$$
\left\|P ^ { q } h \left|\left\|\leq \alpha\left|\|h|| \mid+\beta\| h \| \quad \forall h \in B_{2} .\right.\right.\right.\right.
$$

Then,

(i) The set $\sigma_{1}$ of eigenvalues of $P$ of modulus 1 in $B_{1}$ is a finite group (in particular it contains 1). Each of these eigenvalue has a finite dimensional eigenspace contained inside $B_{2}$.

(ii) There exist in $B_{2}$ finite rank operators $Q_{\lambda}$ for $\lambda \in \sigma$ and an operator $R$ such that $\left\|R^{n} \mid\right\| \leq C \gamma^{n}$ where $C$ is a positive constant and $\left.\gamma \in\right] 0,1[$, and such that for every $\lambda, \lambda^{\prime} \in \sigma_{1}$ we have

$$
\begin{gathered}
Q_{\lambda} \circ Q_{\lambda^{\prime}}=Q_{\lambda} \delta_{\lambda, \lambda^{\prime}}, \quad Q_{\lambda} \circ R=R \circ Q_{\lambda}=0, \\
P=\sum_{\lambda \in \sigma_{1}} \lambda Q_{\lambda}+R .
\end{gathered}
$$

An important consequence of the above result is that the spectrum of $P$ in $B_{2}$ is composed of a finite set of eigenvalues on the unit circle (with finite dimensional eigenspaces) and of another part contained in a disk of radius smaller than 1 .

We now introduce a subspace of $l^{1}(\mathbf{X})$ which will play the role of $B_{2}$ in the above theorem. First define, if $\pi \in l^{1}(\mathbf{X})$,

$$
\bigvee \pi=\sum_{\mathbf{x} \in \mathbf{X}}|\pi(\mathbf{x})| \frac{\nu(\mathbf{x})}{\mathbb{P}(\text { fut }(\mathbf{x}))} .
$$


Now put

$$
\mathrm{BV}(\mathbf{X})=\left\{\pi \in l^{1}(\mathbf{X}) \mid \bigvee \pi<+\infty\right\}
$$

(The reason of the symbol $\mathrm{BV}(\mathbf{X})$ is that this space will play the role of the space of bounded variation functions when the spectral theorem is directly applied to the piecewise affine maps $[10,4])$.

On the subspace $\mathrm{BV}(\mathbf{X})$ we can consider the stronger norm $\||\pi|\|=\|\pi\|_{1}+\bigvee \pi$. We have the following result:

Proposition 2. If $|a|>2$, then, the triple $\left(\left(l^{1}(\mathbf{X}),\|\cdot\|_{1}\right),(\mathrm{BV}(\mathbf{X}),\||\cdot|\|), P\right)$ satisfies the assumption of the Ionescu, Tulcea, and Marinescu theorem.

Proof. In the case when $\mathbf{X}$ is finite, everything becomes trivial; from now on we will thus assume that $\mathbf{X}$ is countable. We now fix a bijection of $\mathbf{X}$ with $\mathbb{N}: n \mapsto \mathbf{x}_{n}$ in such a way that $n \mapsto \nu\left(\mathbf{x}_{n}\right)$ is increasing. Write

$$
\alpha_{k}=\frac{\nu\left(\mathbf{x}_{k}\right)}{\mathbb{P}\left(\text { fut }\left(\mathbf{x}_{k}\right)\right)} .
$$

$\alpha_{k}$ diverges to $+\infty$ for $k \rightarrow+\infty$. If $\pi \in l^{1}(\mathbf{X})$, we can write

$$
\bigvee \pi=\sum_{k=1}^{+\infty}\left|\pi\left(\mathbf{x}_{k}\right)\right| \alpha_{k} .
$$

The fact that $\mathrm{BV}(\mathbf{X})$ is dense inside $l^{1}(\mathbf{X})$ is obvious since sequences in $l^{1}(\mathbf{X})$ which are definitely equal to zero, are clearly in $\mathrm{BV}(\mathbf{X})$. We now prove that the embedding of $\mathrm{BV}(\mathbf{X})$ inside $l^{1}(\mathbf{X})$ is precompact, namely, that any sequence in $\mathrm{BV}(\mathbf{X})$ which is bounded in the norm $\|\cdot\|$, contains a convergent (in the sense of $\|\cdot\|$ ) subsequence. Let $\pi_{n}$ be a sequence in $\operatorname{BV}(\mathbf{X})$ such that $\left\|\left|\pi_{n} \|\right| \leq M\right.$ for all $n$. Since, in particular, $\pi_{n}$ is bounded in the norm $\|\cdot\|$, a standard diagonal technique shows that there exist a subsequence $\pi_{n_{h}}$ converging to some sequence $\pi$, pointwisely, namely, for any $k \in \mathbb{N}$ we have that $\lim _{h \rightarrow+\infty} \pi_{n_{h}}\left(\mathbf{x}_{k}\right) \rightarrow \pi\left(\mathbf{x}_{k}\right)$. From now on, for the sake of simplicity of notation we will denote $n_{h}$ as $n$. It is easy to see that

$$
\bigvee \pi=\sum_{k=1}^{+\infty}\left|\pi\left(\mathbf{x}_{k}\right)\right| \alpha_{k} \leq M
$$

so that, in particular, $\pi \in \mathrm{BV}(\mathbf{X})$. Notice now that

$$
\begin{aligned}
\sum_{k=N}^{+\infty}\left|\pi_{n}\left(\mathbf{x}_{k}\right)-\pi\left(\mathbf{x}_{k}\right)\right| & =\sum_{k=N}^{+\infty} \frac{\left|\pi_{n}\left(\mathbf{x}_{k}\right)-\pi\left(\mathbf{x}_{k}\right)\right| \alpha_{k}}{\alpha_{k}} \\
& \leq\left[\sup _{k \geq N} \frac{1}{\alpha_{k}}\right] \sum_{k=N}^{+\infty}\left|\pi_{n}\left(\mathbf{x}_{k}\right)-\pi\left(\mathbf{x}_{k}\right)\right| \alpha_{k} \\
& \leq\left[\sup _{k \geq N} \frac{1}{\alpha_{k}}\right] 2 M
\end{aligned}
$$


Fix now $\epsilon>0$ and choose $N$ in such a way that the last term in (12) is smaller than $\epsilon / 2$. Then choose $n_{0} \in \mathbb{N}$ such that

$$
\sum_{k=1}^{N-1}\left|\pi_{n}\left(\mathbf{x}_{k}\right)-\pi\left(\mathbf{x}_{k}\right)\right|<\epsilon / 2 \quad \forall n \geq n_{0} .
$$

This implies that

$$
\sum_{k=1}^{+\infty}\left|\pi_{n}\left(\mathbf{x}_{k}\right)-\pi\left(\mathbf{x}_{k}\right)\right|<\epsilon \quad \forall n \geq n_{0} .
$$

Finally we need to prove inequality (11). Notice that

$$
P_{\mathbf{x y}}^{q}=t_{\mathbf{x y}}^{q} \frac{\mathbb{P}(\text { fut }(\mathbf{y}))}{|a|^{q} \mathbb{P}(\text { fut }(\mathbf{x}))} .
$$

Let $\pi \in \mathrm{BV}(\mathbf{X})$. We have that

$$
\begin{aligned}
\bigvee P \pi & =\sum_{\mathbf{x} \in \mathbf{X}}|(P \pi)(\mathbf{x})| \frac{\nu(\mathbf{x})}{\mathbb{P}(\text { fut }(\mathbf{x}))} \\
& \leq \sum_{\mathbf{x} \in \mathbf{X}} \sum_{\mathbf{y} \in \mathbf{X}}|\pi(\mathbf{y})| t_{\mathbf{y} \mathbf{x}} \frac{\mathbb{P}(f u t(\mathbf{x}))}{|a| \mathbb{P}(\text { fut }(\mathbf{y}))} \frac{\nu(\mathbf{x})}{\mathbb{P}(\text { fut }(\mathbf{x}))} \\
& =\frac{1}{|a|} \sum_{\mathbf{y} \in \mathbf{X}} \frac{|\pi(\mathbf{y})|}{\mathbb{P}(\text { fut }(\mathbf{y}))} \sum_{\mathbf{x} \in \mathcal{F}(\mathbf{y})} \nu(\mathbf{x}),
\end{aligned}
$$

where

$$
\mathcal{F}(\mathbf{y})=\left\{\mathbf{x} \in \mathbf{X}: t_{\mathbf{y x}}=1\right\}
$$

Put

$$
\delta=\min _{A \in \mathcal{I}} \mathbb{P}(A)
$$

and recall that $N$ is the cardinality of $\mathcal{I}$. Fix moreover $\nu \in \mathbb{N}$ and define

$$
\begin{aligned}
& \mathbf{X}^{o}=\{\mathbf{y} \in \mathbf{X} \mid \nu(\mathbf{y}) \leq \nu\} \\
& \mathbf{X}^{\prime}=\left\{\mathbf{y} \in \mathbf{X} \backslash \mathbf{X}^{o}|\mathbb{P}(\operatorname{fut}(\mathbf{y}))<\delta /| a \mid\right\} \\
& \mathbf{X}^{\prime \prime}=\left\{\mathbf{y} \in \mathbf{X} \backslash \mathbf{X}^{o}|\mathbb{P}(\operatorname{fut}(\mathbf{y})) \geq \delta /| a \mid\right\}
\end{aligned}
$$

Notice now that all the elements $\mathbf{x} \in \mathcal{F}(\mathbf{y})$ will satisfy the condition $\nu(\mathbf{x})=1$ with exception of utmost two of them for which it will however hold $\nu(\mathbf{x}) \leq \nu(\mathbf{y})+1$. Moreover, if $\mathbf{y} \in \mathbf{X}^{\prime}$, then, $|\mathcal{F}(\mathbf{y})| \leq 2$. With these considerations we can now continue 
the estimation (13):

$$
\begin{aligned}
& \frac{1}{|a|} \sum_{\mathbf{y} \in \mathbf{X}} \frac{|\pi(\mathbf{y})|}{\mathbb{P}(\text { fut }(\mathbf{y}))} \sum_{\mathbf{x} \in \mathcal{F}(\mathbf{y})} \nu(\mathbf{x}) \\
=\frac{1}{|a|} & {\left[\sum_{\mathbf{y} \in \mathbf{X}^{o}} \frac{|\pi(\mathbf{y})|}{\mathbb{P}(\text { fut }(\mathbf{y}))} \sum_{\mathbf{x} \in \mathcal{F}(\mathbf{y})} \nu(\mathbf{x})+\sum_{\mathbf{y} \in \mathbf{X}^{\prime}} \frac{|\pi(\mathbf{y})|}{\mathbb{P}(\text { fut }(\mathbf{y}))} \sum_{\mathbf{x} \in \mathcal{F}(\mathbf{y})} \nu(\mathbf{x})\right.} \\
& \left.+\sum_{\mathbf{y} \in \mathbf{X}^{\prime \prime}} \frac{|\pi(\mathbf{y})|}{\mathbb{P}(\text { fut }(\mathbf{y}))} \sum_{\mathbf{x} \in \mathcal{F}(\mathbf{y})} \nu(\mathbf{x})\right] \\
\leq & \frac{1}{|a|}\left[N(\nu+1) \max _{\mathbf{y} \in \mathcal{X}_{I}^{o}} \frac{1}{\mathbb{P}(\text { fut }(\mathbf{y}))} \sum_{\mathbf{y} \in \mathbf{X}^{o}}|\pi(\mathbf{y})|+\sum_{\mathbf{y} \in \mathbf{X}^{\prime}} \frac{|\pi(\mathbf{y})|}{\mathbb{P}(\text { fut }(\mathbf{y}))}(2 \nu(\mathbf{y})+2)\right] \\
+ & \frac{1}{|a|}\left[\sum_{\mathbf{y} \in \mathbf{X}^{\prime \prime}} \frac{|\pi(\mathbf{y})|}{\mathbb{P}(\text { fut }(\mathbf{y}))}(2 \nu(\mathbf{y})+2)+\frac{|a|}{\delta} N \frac{\left.\sum_{\mathbf{y} \in \mathbf{X}^{\prime \prime}}|\pi(\mathbf{y})|\right]}{\leq} \frac{2(1+1 / \nu)}{|a|} \bigvee(\pi)+N\left(\frac{1}{\delta}+\frac{\nu+1}{|a|} \max _{\mathbf{y} \in \mathbf{X}^{o}} \frac{1}{\mathbb{P}(\text { fut }(\mathbf{y}))}\right)\|\pi\|_{1}\right. \\
= & \frac{2(1+1 / \nu)}{|a|}\||\pi|\|+N\left(\frac{1}{\delta}+\frac{\nu+1}{|a|} \max _{\mathbf{y} \in \mathbf{X}^{o}} \frac{1}{\mathbb{P}(\text { fut }(\mathbf{y}))}\right)\|\pi\|_{1} .
\end{aligned}
$$

To complete the proof it is now sufficient to notice that since $|a|>2$, then there exists $\nu \in \mathbb{N}$ such that

$$
\frac{2(1+1 / \nu)}{|a|}<1
$$

In the case when 1 is a simple eigenvalue and there is no other eigenvalue on the unit circle, the decomposition of the transition probability operator $P$ assumes a particular simple form. The projection operator $Q_{1}$ on the subspace generated by $\pi_{0}$ takes the form $Q_{1} \pi=(\pi \cdot e) \pi_{0}$. We thus have

$$
P=Q_{1}+R
$$

where

$$
\left\|\left|R^{n}\right|\right\| \leq C \gamma^{n}
$$

for some $C \geq 0$ and $\gamma \in[0,1[$. This implies that, if $\pi \in \mathrm{BV}(\mathbf{X})$, we have that

$$
\text { ||| } P^{n} \pi-Q_{1} \pi||\left|\leq C \gamma^{n}\right||| \pi \mid \|,
$$

namely, exponential convergence to the invariant measure.

Non-cyclicity is the property needed to have such a spectral structure:

Proposition 3. Assume that the Markov chain is non-cyclic. Then, there exists exactly one $\pi_{0} \in \mathrm{BV}(\mathbf{X})$ which is a probability measure and such that $P \pi_{0}=\pi_{0}$. Moreover, $P$ does not have any further eigenvalue on the unit circle. 
Proof. First part follows from previous considerations on non-cyclic Markov chains. To prove the last assertion, recall that $P^{n} \pi \rightarrow Q_{1} \pi$ in norm $l^{1}$ for every $\pi \in l^{1}(\mathbf{X})$. This immediately shows that there can not be either eigenvalues of norm equal to 1 .

4. Expected entrance times for non-cyclic Markov chains. Consider the Markov chain $(P, \mathbf{X})$ associated with $(\Gamma, \mathcal{I})$. Assume the Markov chain to be noncyclic. Let $\pi \in \mathrm{BV}(\mathbf{X})$ and let $X_{n}=X_{n}^{\pi}$ be the Markov process on the set of states $\mathbf{X}$ with initial distribution $\pi$ as defined above. Let $\overline{\mathbf{x}} \in \mathbf{X}$ be a fixed target state. We want to give estimations of $\mathbb{E}_{\pi}\left(T_{\overline{\mathbf{x}}}\right)$.

It follows from Proposition 3 and previous considerations that there exists a onedimensional projection operator $Q_{1}: l^{1}(\mathbf{X}) \rightarrow l^{1}(\mathbf{X})$ defined by $Q_{1} \pi=(\pi \cdot e) \pi_{o}$ where $\pi_{0}$ is the only invariant probability measure for $P$ (with $\pi_{0}(\mathbf{x})>0$ for every $\mathbf{x} \in \mathbf{X}$ ), numbers $C>0$ and $\gamma \in(0,1)$ such that for every $\pi \in \mathrm{BV}(\mathbf{X})$ we have

$$
\left\|\left|P^{n} \pi-Q_{1} \pi\right||| \leq C \gamma^{n}|\|\pi \mid\| \quad \forall n \in \mathbb{N} .\right.
$$

Standard considerations show that, if $\pi \in \mathrm{BV}(\mathbf{X})$, then

$$
\mathbb{E}_{\pi}\left(T_{\overline{\mathbf{x}}}\right)=\frac{\sum_{n=0}^{+\infty}\left[P_{\overline{\mathbf{x} x}}^{n}-\pi_{0}(\overline{\mathbf{x}})\right]-\sum_{n=0}^{+\infty}\left[(P \pi)_{\overline{\mathbf{x}}}^{n}-\pi_{0}(\overline{\mathbf{x}})\right]}{\pi_{0}(\overline{\mathbf{x}})} .
$$

It follows from (16) that the numerator of (17) can be bounded above by

$$
\frac{c}{1-\gamma}\left(||\left|\delta_{\overline{\mathbf{x}}}\right|||+|||\pi|||\right) \text {. }
$$

On the other hand, we have that

$$
\left\|\left|\delta_{\overline{\mathbf{x}}} \|\right| \leq \frac{\nu(\mathbf{x})}{\mathbb{P}(\text { futx })}+1\right.
$$

(we recall that $\delta_{\overline{\mathbf{x}}}$ is the probability measure concentrated on the state $\mathbf{x}$ ). We thus obtain

$$
\mathbb{E}_{\pi}\left(T_{\overline{\mathbf{x}}}\right) \leq \frac{\frac{c}{1-\gamma}\left[\frac{\nu(\mathbf{x})}{\mathbb{P}(\text { fut }(\mathbf{x})}+1+\||| \pi \mid\|\right]}{\pi_{0}(\mathbf{x})} .
$$

The above formula presents a number of problems: in particular, it is not clear if the numerator can be bounded by a constant. To overcome these difficulties we will take a slightly different road and we will work with the family of Markov chains associated with the family of partitions $\mathcal{I}^{r+1}$ and we will concentrate on particular subsets of their respective principal states. It can be shown that the set of states $\mathbf{X}_{\mathcal{I}^{r+1}}$ can be identified with the subset of $\mathbf{X}_{\mathcal{I}}^{r+1}$ consisting of the $(r+1)$-uples $\left(\mathbf{x}_{\mathbf{0}}, \ldots, \mathbf{x}_{r}\right)$ such that the word $\mathbf{x}_{0} \cdots \mathbf{x}_{r} \in \Sigma^{*}(\Gamma, \mathcal{I})$, equivalently such that

$$
\text { fut }\left(\mathbf{x}_{0}\right) \cap \Gamma^{-1}\left(\text { fut }\left(\mathbf{x}_{1}\right) \cap \cdots \cap \Gamma^{-r}\left(\text { fut }\left(\mathbf{x}_{r}\right) \neq \emptyset\right)\right) .
$$


Moreover, we have that

$$
\text { fut }\left(\mathbf{x}_{0}, \ldots, \mathbf{x}_{r}\right)=\operatorname{fut}\left(\mathbf{x}_{0}\right) \cap \Gamma^{-1}\left(\operatorname{fut}\left(\mathbf{x}_{1}\right)\right) \cap \cdots \cap \Gamma^{-r}\left(\text { fut }\left(\mathbf{x}_{r}\right)\right) .
$$

The transition probability is given by

$$
P_{\left(\mathbf{x}_{0}, \ldots, \mathbf{x}_{r}\right),\left(\mathbf{y}_{0}, \ldots, \mathbf{y}_{r}\right)}= \begin{cases}P_{\mathbf{y}_{r} \mathbf{x}_{r}} & \text { if } \mathbf{x}_{k}=\mathbf{y}_{k+1} k=0, \ldots, r-1 \\ 0 & \text { otherwise. }\end{cases}
$$

It is a standard fact that this new Markov chain is still non-cyclic. If $\pi \in l^{1}(\mathbf{X})$, define $\pi^{(r)} \in l^{1}\left(\mathbf{X}^{r+1}\right)$ by

$$
\pi^{(r)}\left(\mathbf{x}_{0}, \ldots, \mathbf{x}_{r}\right)=\pi\left(\mathbf{x}_{0}\right) P_{\mathbf{x}_{0} \mathbf{x}_{1}} P_{\mathbf{x}_{1} \mathbf{x}_{2}} \cdots P_{\mathbf{x}_{r-1} \mathbf{x}_{r}} .
$$

It is easy to see that the unique invariant probability measure is given by $\pi_{0}^{(r)}$. Moreover, we have that

$$
\mathrm{BV}\left(\mathbf{X}^{r+1}\right)=\left\{\pi^{(r)} \in l^{1}\left(\mathbf{X}^{r+1}\right) \mid \pi \in \mathrm{BV}(\mathbf{X})\right\} .
$$

Fix now as initial probability measure $\pi^{(r)}$. The associated Markovian process $X_{n}^{\pi^{(r)}}$ is simply the $(r+1)$-th power of the Markovian process $X_{n}$. Namely,

$$
X_{n}^{\pi^{(r)}}=\left(X_{n}^{\pi}, X_{n+1}^{\pi}, \ldots, X_{n+r}^{\pi}\right) .
$$

Fix

$$
\overline{\mathbf{x}}=\left(\overline{\mathbf{x}}_{0}, \overline{\mathbf{x}}_{1}, \cdots, \overline{\mathbf{x}}_{r}\right) \in \mathbf{X}^{r+1} .
$$

We will still have exponential convergence to the invariant probability measure so that we can use the same formula (17) to calculate the expected entrance time of the Markov process $X_{n}^{\pi^{(r)}}$ in the state $\overline{\mathbf{x}}$. We obtain

$$
\mathbb{E}_{\pi^{(r)}}\left(T_{\overline{\mathbf{x}}}\right)=\frac{\left.\sum_{n=0}^{+\infty}\left[\left(P^{(r)}\right)_{\overline{\mathbf{x}}}^{n}-\pi_{0}^{(r)}(\overline{\mathbf{x}})\right]-\sum_{n=0}^{+\infty}\left[\left(P^{(r)}\right)^{n} \pi^{(r)}\right)_{\overline{\mathbf{x}}}-\pi_{0}^{(r)}(\overline{\mathbf{x}})\right]}{\pi_{0}^{(r)}(\overline{\mathbf{x}})} .
$$

Simple algebraic manipulation permits to transform (19) into

$$
\begin{aligned}
\mathbb{E}_{\pi^{(r)}}\left(T_{\overline{\mathbf{x}}}\right)= & \frac{\left.\sum_{n=0}^{r-1}\left[\left(P^{(r)}\right)_{\overline{\mathbf{x}}}^{n}-\pi_{0}^{(r)}(\overline{\mathbf{x}})\right]-\sum_{n=0}^{r-1}\left[\left(P^{(r)}\right)^{n} \pi^{(r)}\right)_{\overline{\mathbf{x}}}-\pi_{0}^{(r)}(\overline{\mathbf{x}})\right]}{\pi_{0}^{(r)}(\overline{\mathbf{x}})}+ \\
+ & \frac{\sum_{n=0}^{+\infty}\left[P_{\overline{\mathbf{x}}_{r} \overline{\mathbf{x}}_{0}}^{n}-\pi_{0}\left(\overline{\mathbf{x}}_{0}\right)\right]-\sum_{n=0}^{+\infty}\left[\left(P^{n} \pi\right)_{\overline{\mathbf{x}}_{0}}-\pi_{0}\left(\overline{\mathbf{x}}_{0}\right)\right]}{\pi_{0}\left(\overline{\mathbf{x}}_{0}\right)} \\
\leq & \frac{\sum_{n=0}^{r-1}\left(P^{(r)}\right)_{\overline{\mathbf{x x}}}^{n}}{\pi_{0}^{(r)}(\overline{\mathbf{x}})}+\frac{\sum_{n=0}^{+\infty}\left[P_{\overline{\mathbf{x}}_{r} \overline{\mathbf{x}}_{0}}^{n}-\pi_{0}\left(\overline{\mathbf{x}}_{0}\right)\right]-\sum_{n=0}^{+\infty}\left[\left(P^{n} \pi\right)_{\overline{\mathbf{x}}_{0}}-\pi_{0}\left(\overline{\mathbf{x}}_{0}\right)\right]}{\pi_{0}\left(\overline{\mathbf{x}}_{0}\right)} .
\end{aligned}
$$


We will assume that the state $\overline{\mathbf{x}}$ belongs to the following subclass of principal states:

$$
\mathbf{X}_{P}^{(r+1)}=\left\{\left(\overline{\mathbf{x}}_{0}, \overline{\mathbf{x}}_{1}, \cdots, \overline{\mathbf{x}}_{r}\right) \in \mathbf{X}^{r+1} \mid \overline{\mathbf{x}}_{0}, \overline{\mathbf{x}}_{r} \in \mathbf{X}_{P}, P_{\overline{\mathbf{x}}_{h}, \overline{\mathbf{x}}_{h+1}}>0 h=0, \ldots, r-1\right\} .
$$

Define

$$
\delta_{1}=\min \left\{\pi_{0}\left(\overline{\mathbf{x}}_{0}\right) \mid \overline{\mathbf{x}}_{0} \in \mathbf{X}_{P}\right\} \quad \delta_{2}=\min \left\{\mathbb{P}\left(\text { fut }\left(\overline{\mathbf{x}}_{0}\right)\right) \mid \overline{\mathbf{x}}_{0} \in \mathbf{X}_{P}\right\}
$$

We then obtain, using (16), that the second fraction in (20) can be bounded by a constant only depending on $\pi$ as follows

$$
\left|\frac{\sum_{n=0}^{+\infty}\left[P_{\overline{\mathbf{x}}_{r} \overline{\mathbf{x}}_{0}}^{n}-\pi_{0}\left(\overline{\mathbf{x}}_{0}\right)\right]-\sum_{n=0}^{+\infty}\left[\left(P^{n} \pi\right)_{\overline{\mathbf{x}}_{0}}-\pi_{0}\left(\overline{\mathbf{x}}_{0}\right)\right]}{\pi_{0}\left(\overline{\mathbf{x}}_{0}\right)}\right| \leq \frac{C}{\delta_{1}(1-\gamma)}\left[\delta_{1}^{-1}+1+\||\pi|||\right] .
$$

It remains to be shown that the numerator of the first fraction can also be bounded by a constant. In order to see this, we first need to develop some combinatorics. Given a vector $\left(\overline{\mathbf{x}}_{0}, \overline{\mathbf{x}}_{1}, \cdots, \overline{\mathbf{x}}_{r}\right) \in \mathbf{X}^{r+1}$, we define the set of its periods as

$$
\mathcal{Q}\left(\overline{\mathbf{x}}_{0}, \overline{\mathbf{x}}_{1}, \cdots, \overline{\mathbf{x}}_{r}\right)=\left\{n \in \mathbb{N} \backslash\{0\} ; \mid \overline{\mathbf{x}}_{n+k}=\overline{\mathbf{x}}_{k}, \quad k=0, \ldots, r-n\right\} .
$$

If $\mathcal{Q}\left(\overline{\mathbf{x}}_{0}, \overline{\mathbf{x}}_{1}, \cdots, \overline{\mathbf{x}}_{r}\right)$ is empty, we will say that the vector is aperiodic. If it is not empty, we define the first principal period as

$$
\bar{n}_{1}=\min \mathcal{Q}\left(\overline{\mathbf{x}}_{0}, \overline{\mathbf{x}}_{1}, \cdots, \overline{\mathbf{x}}_{r}\right) .
$$

A vector having principal period equal to $\bar{n}_{1}$ consists of $s_{1}$ equal consecutive blocks of length $\bar{n}_{1}$ :

$$
\left(\overline{\mathbf{x}}_{0}, \overline{\mathbf{x}}_{1}, \cdots, \overline{\mathbf{x}}_{\bar{n}_{1}-1}\right)
$$

and an eventual final one of length $r_{1}<\bar{n}_{1}$ :

$$
\left(\overline{\mathbf{x}}_{0}, \overline{\mathbf{x}}_{1}, \cdots, \overline{\mathbf{x}}_{r_{1}-1}\right) \text {. }
$$

We thus have

$$
r=s_{1} \bar{n}_{1}+r_{1}, \quad r_{1}<\bar{n}_{1}
$$

Clearly,

$$
\left\{\bar{n}_{1}, 2 \bar{n}_{1}, \ldots, s_{1} \bar{n}_{1}\right\} \subseteq \mathcal{Q}\left(\overline{\mathbf{x}}_{0}, \overline{\mathbf{x}}_{1}, \cdots, \overline{\mathbf{x}}_{r}\right)
$$

Consider now the last block (22) and let $\bar{n}_{2}$ be its first principal period. We will have

$$
r_{1}=s_{2} \bar{n}_{2}+r_{2}, \quad r_{2}<\bar{n}_{2} .
$$


Going on in this way, we finally determine positive integers, $\bar{n}_{1}, \bar{n}_{2}, \ldots, \bar{n}_{l}$ (called principal periods) and $s_{1}, s_{2}, \ldots, s_{l}$ called multiplicities) having the following properties:

$$
\begin{gathered}
0 \leq r-\sum_{j=1}^{k} s_{j} \bar{n}_{j}<\bar{n}_{k}, \quad k=1, \ldots l \\
\mathcal{Q}\left(\overline{\mathbf{x}}_{0}, \overline{\mathbf{x}}_{1}, \cdots, \overline{\mathbf{x}}_{r}\right)=\left\{\sum_{j=1}^{k-1} s_{j} \bar{n}_{j}+s \bar{n}_{k} \mid 1 \leq k \leq l \quad 0 \leq j \leq s_{k}\right\} .
\end{gathered}
$$

Notice that in (24) for $k=l$ we may have equality or strict inequality: this last case happen when, following the procedure above illustrated, at a certain point we end up with an aperiodic block. We are now ready to begin the estimation of the numerator of the first addend of (20). Assume that $\overline{\mathbf{x}}=\left(\overline{\mathbf{x}}_{0}, \overline{\mathbf{x}}_{1}, \cdots, \overline{\mathbf{x}}_{r}\right)$ has principal vectors $\bar{n}_{1}, \bar{n}_{2}, \ldots, \bar{n}_{l}$ and multiplicities $s_{1}, s_{2}, \ldots, s_{l}$. We then have

$$
\left.\sum_{n=0}^{r-1}\left(P^{(r)}\right)_{\overline{\mathbf{x} x}}^{n}=\sum_{n \in \mathcal{P}}\left(P^{(r)}\right)_{\overline{\mathbf{x} x}}^{n}=\sum_{k=1}^{l} \sum_{j=0}^{s_{k}-1}\left(P^{(r)}\right)_{\overline{\mathbf{x x}}}^{\left(\sum_{i=1}^{k-1} s_{i} \bar{n}_{i}+j \bar{n}_{k}\right.}\right) .
$$

We now need to estimate the right hand side inner summation. Notice that, if $j>0$,

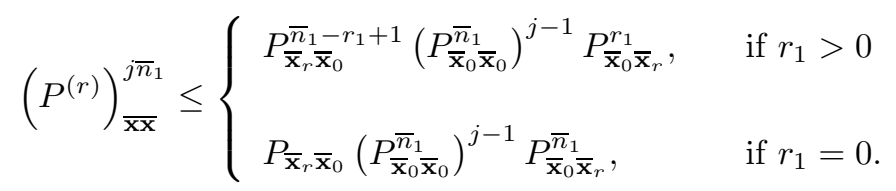

More generally, we obtain, if $j>0$,

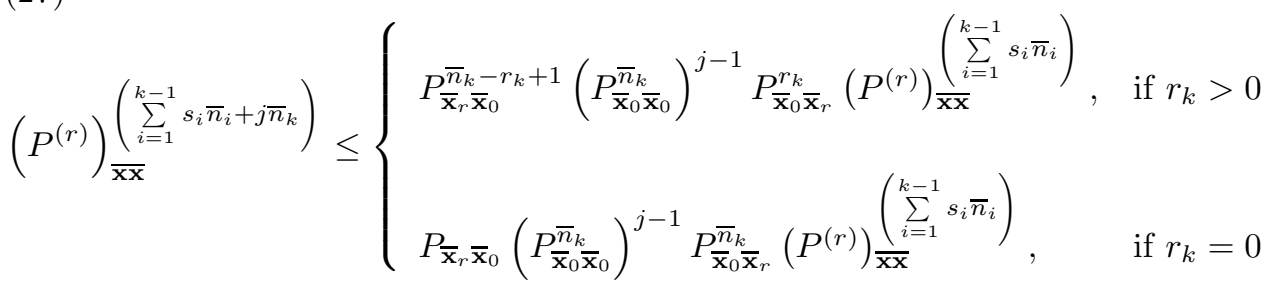

Therefore,

$$
\sum_{j=0}^{s_{k}-1}\left(P^{(r)}\right)_{\overline{\mathbf{x} x}}^{\left(\sum_{i=1}^{k-1} s_{i} \bar{n}_{i}+j \bar{n}_{k}\right)} \leq\left[\alpha_{k} \sum_{j=0}^{s_{k}-2}\left(P_{\mathbf{x}_{0} \overline{\mathbf{x}}_{0}}^{\bar{n}_{k}}\right)^{j}+1\right]\left(P^{(r)}\right)_{\overline{\mathbf{x x}}}^{\left(\sum_{i=1}^{k-1} s_{i} \bar{n}_{i}\right)},
$$

where

$$
\alpha_{k}= \begin{cases}P_{\overline{\mathbf{x}}_{r} \overline{\mathbf{x}}_{0}}^{\bar{n}_{k}-r_{k}+1} P_{\overline{\mathbf{x}}_{0} \overline{\mathbf{x}}_{r}}^{r_{k}}, & \text { if } r_{k}>0 \\ P_{\overline{\mathbf{x}}_{r} \overline{\mathbf{x}}_{0}} P_{\overline{\mathbf{x}}_{0} \overline{\mathbf{x}}_{r}}^{\bar{n}_{k},} & \text { if } r_{k}=0 .\end{cases}
$$


In any case we can estimate $\alpha_{k}$ as follows

$$
\alpha_{k} \leq P_{\overline{\mathbf{x}}_{0} \overline{\mathbf{x}}_{0}}^{\bar{n}_{k}+1}
$$

By induction, using (25) and (28), we thus obtain

$$
\begin{aligned}
\sum_{n=0}^{r-1}\left(P^{(r)}\right)_{\overline{\mathbf{x}}}^{n} & \leq\left[1+\alpha_{1} \sum_{j=0}^{s_{1}-2}\left(P_{\overline{\mathbf{x}}_{0} \overline{\mathbf{x}}_{0}}^{\bar{n}_{\bar{x}}}\right)^{j}\right. \\
& \left.+\alpha_{1}\left(P_{\overline{\mathbf{x}}_{0} \overline{\mathbf{x}}_{0}}^{\bar{n}_{1}}\right)^{s_{1}-1}\left[1+\alpha_{2} \sum_{j=0}^{s_{2}-2}\left(P_{\overline{\mathbf{x}}_{0} \overline{\mathbf{x}}_{0}}^{\bar{n}_{2}}\right)^{j}+\alpha_{2}\left(P_{\overline{\mathbf{x}}_{0} \overline{\mathbf{x}}_{0}}^{\overline{\mathbf{n}}_{0}}\right)^{s_{2}-1}[\cdots]\right]\right] .
\end{aligned}
$$

We now make use the following simple lemma.

Lemma 1. There exists $q \in(0,1)$ such that

$$
P_{\overline{\mathbf{x}}_{0}, \overline{\mathbf{x}}_{0}}^{n}<q, \quad \forall n \geq 1, \forall \overline{\mathbf{x}}_{0} \in \mathbf{X}_{P} .
$$

Proof. Notice that $P_{\overline{\mathbf{x}}_{0}, \overline{\mathbf{x}}_{0}}^{n} \rightarrow \pi_{0}\left(\overline{\mathbf{x}}_{0}\right)<1$ for any $\overline{\mathbf{x}}_{0} \in \mathbf{X}_{P}$. Hence, if we fix

$$
q \in\left(\max \left\{\pi_{0}(\overline{\mathbf{x}}) \mid \overline{\mathbf{x}} \in \mathbf{X}_{P}\right\}, 1\right)
$$

we have that, if $n$ is sufficiently large,

$$
P_{\overline{\mathbf{x}}_{0}, \overline{\mathbf{x}}_{0}}^{n}<q \quad \forall \overline{\mathbf{x}}_{0} \in \mathbf{X}_{P} .
$$

Notice now that if $P_{\overline{\mathbf{x}}_{0}, \overline{\mathbf{x}}_{0}}^{n}=1$ for some $i$ and some $n \geq 1$, then, $P_{\overline{\mathbf{x}}_{0}, \overline{\mathbf{x}}_{0}}^{k n}=1$ for every $k \in \mathbb{N}$ and this is impossible for previous considerations. This implies the result.

We are now ready to prove the key result.

Proposition 4.

$$
\sum_{n=0}^{r-1}\left(P^{(r)}\right)_{\overline{\mathbf{x x}}}^{n} \leq(1-q)^{-1}, \quad \forall \overline{\mathbf{x}} \in \mathbf{X}_{P}^{(r)} .
$$

Proof. Since $\alpha_{k}<q$ and $P_{\overline{\mathbf{x}}_{0} \overline{\mathbf{x}}_{0}}^{\bar{n}_{k}}<q$ for every $k$, it follows from (31) that

$$
\sum_{n=0}^{r-1}\left(P^{(r)}\right)_{\overline{\mathbf{x}} \mathbf{x}}^{n} \leq \sum_{j=0}^{s_{1}-1} q^{j}+q^{s_{1}}\left(\sum_{j=0}^{s_{2}-1} q^{j}+q^{s_{2}}[\cdot]\right) .
$$

It remains to be shown that the above right hand side expression in $q$ is smaller than $(1-q)^{-1}$. This can be proven by induction on $l$. Call $\beta\left(s_{1}, \cdots, s_{l}\right)$ the above expression. If $l=1$ we have that

$$
\beta\left(s_{1}\right)=\sum_{j=0}^{s_{1}} \leq(1-q)^{-1}
$$

Assume it to hold for $l-1$ and notice that

$$
\beta\left(s_{1}, \cdots, s_{l}\right)=\sum_{j=0}^{s_{1}-1} q^{j}+q^{s_{1}} \beta\left(s_{2}, \cdots, s_{l}\right) \leq \frac{1-q^{s_{k}}}{1-q}+q^{s_{k}} \frac{1}{1-q}=\frac{1}{1-q} .
$$


We thus obtain the following result.

Corollary 1. For every $\pi \in \mathrm{BV}(\mathbf{X})$, there exist constants $A, B>0$ such that

$$
\mathbb{E}_{\pi^{(r)}}\left(T_{\overline{\mathbf{x}}}\right) \leq \frac{A}{\pi_{0}^{(r)}(\overline{\mathbf{x}})}+B
$$

for every $\overline{\mathbf{x}} \in \mathbf{X}_{P}^{(r)}$ and for every $r$. Moreover, $A$ can be chosen to be independent of $\pi$, while $B$ can be chosen of the type $B=B_{1}+B_{2}\left\||\| \pi| \mid\right.$ with $B_{1}$ and $B_{2}$ constants independent of $\pi$.

4.1. Proof of the main Theorem. As a concrete application of the bound we have found, we now prove Theorem 1.

Consider the piecewise affine map $\Gamma$ on $[-1,1]$ defined in $(5)$. Let $(\mathbf{X}, P)$ be the Markov chain associated with $\Gamma$ and with the canonical partition. The principal states $<I_{k}>$ with $|k| \leq k_{o}$ will be called central states; notice that from any central state there is a non-zero probability edge to any other principal state.

We now prove the following result.

Proposition 5. If $|a|>2$, the Markov chain $(\mathbf{X}, P)$ associated with $\Gamma$ on $[-1,1]$ and with the canonical partition is non-cyclic.

Proof. First, we show that given any $\mathbf{x}_{1} \in \mathbf{X}$, there is a non-zero probability path in the direct graph on $\mathbf{X}$ which connects the state $\mathbf{x}_{1}$ to a central state. Notice that if there are more than two edges exiting $\mathbf{x}_{1}$, then, from the one-dimensional structure of $\Gamma$, it follows that one of them will surely lead to a central state. If there are one or two exiting edges, then for sure we can find a state $\mathbf{x}_{2}$ such that $\mathbf{x}_{1} \mathbf{x}_{2}$ is a possible path and fut $\left(\mathbf{x}_{2}\right) \geq(|a| / 2)$ fut $\left(\mathbf{x}_{1}\right)$. Since $|a|>2$, it easily follows, by an inductive argument, that there must be a path in the graph $\mathbf{x}_{1} \mathbf{x}_{2} \cdots \mathbf{x}_{n}$ with $\mathbf{x}_{n}$ central state. Notice now that, since from $\mathbf{x}_{n}$ we can reach in one step every other principal state and since by definition any state is reachable in a finite number of steps by some principal state it follows that the chain is indecomposable. Moreover, since $P_{\mathbf{x}_{n}, \mathbf{x}_{n}}^{k}>0$ for every $k \in \mathbb{N}$ and there exists $m \in \mathbb{N}$ such that $P_{\mathbf{x}_{n}, \mathbf{x}_{1}}^{k}>0$. We obtain that $P_{\mathbf{x}_{1}, \mathbf{x}_{1}}^{k}>0$ for every $k \geq n+m$. To prove that the chain is non-cyclic it only remains to be proven that it is positively recurrent. Since, invariant probability measures surely exist, it is actually sufficient to prove that it is recurrent. This will be done by showing that $N_{\mathbf{x}, \mathbf{y}}=+\infty$ for any pair of states $\mathbf{x}, \mathbf{y} \in \mathbf{X}$ (we recall that the matrix $N$ has been defined in Section 3). Recall the decomposition

$$
P=\sum_{\lambda \in \sigma_{1}} \lambda Q_{\lambda}+R
$$

Since $\sigma_{1}$ is a finite group, we have that there exists $n_{0} \in \mathbb{N}$ such that

$$
P^{k n_{0}}=Q+R^{k n_{0}}, \quad \forall k \in \mathbb{N},
$$


where we have posed

$$
Q=\sum_{\lambda \in \sigma_{1}} Q_{\lambda}
$$

Fix any pair of states $\mathbf{x}_{0}, \mathbf{y}_{0} \in \mathbf{X}$ such that $Q_{\mathbf{x}_{0}, \mathbf{y}_{0}} \neq 0$. We thus obtain

$$
\lim _{n \rightarrow+\infty} \sum_{k=0}^{n} P_{\mathbf{x}_{0}, \mathbf{y}_{0}}^{k n_{0}}=\lim _{n \rightarrow+\infty}\left[(n+1) Q_{\mathbf{x}_{0}, \mathbf{y}_{0}}+R_{\mathbf{x}_{0}, \mathbf{y}_{0}}^{k n_{0}}\right]=\infty .
$$

This implies that necessarily

$$
N_{\mathbf{x}_{0}, \mathbf{y}_{0}}=\lim _{n \rightarrow+\infty} \sum_{k=0}^{n} P_{\mathbf{x}_{0}, \mathbf{y}_{0}}^{k} \geq \lim _{n \rightarrow+\infty} \sum_{k=0}^{n} P_{\mathbf{x}_{0}, \mathbf{y}_{0}}^{k n_{0}}=+\infty .
$$

Hence, $N_{\mathbf{x}_{0}, \mathbf{y}_{0}}=+\infty$. Since the chain is indecomposable, it follows that $N_{\mathbf{x}, \mathbf{y}}=+\infty$ for any $\mathbf{x}, \mathbf{y} \in \mathbf{X}$. This completes the proof.

Consider now the principal state $\left\langle I_{0}>\right.$ and notice that $\Gamma I_{0} \supseteq I_{0}$. Hence, the $(r+1)$-uple $<I_{0}><I_{0}>\cdots<I_{0}>$ is in $\mathbf{X}_{P}^{(r+1)}$. Notice moreover, that

$$
\begin{aligned}
\operatorname{fut}\left(<I_{0}><I_{0}>\cdots<I_{0}>\right) & =I_{0} \cap \Gamma^{-1}\left(I_{0}\right) \cap \cdots \cap \Gamma^{-r}\left(I_{0}\right) \\
& =\left(-|a|^{-(r+1)},|a|^{-(r+1)}\right) .
\end{aligned}
$$

Using Corollary 1, we obtain

$$
\begin{aligned}
\mathbb{E}\left(T_{\left(-|a|^{-(r+1)},|a|^{-(r+1)}\right)}\right) & \leq \mathbb{E}_{\pi_{\mathrm{u}}^{(r)}}\left(T_{<I_{0}><I_{0}>\cdots<I_{0}>}\right) \leq \frac{A}{\pi_{0}^{(r)}\left(<I_{0}><I_{0}>\cdots<I_{0}>\right)}+B \\
& =\frac{A}{\pi_{0}\left(<I_{0}>\right)\left(P_{\left.<I_{0}><I_{0}>\right)^{r}}\right.}+B=\frac{A}{\pi_{0}\left(<I_{0}>\right)|a|^{-r}}+B .
\end{aligned}
$$

Fix now any $\epsilon>0$ and choose

$$
r=\left\lfloor\frac{\ln \epsilon^{-1}}{\ln |a|}\right\rfloor
$$

Clearly, $\left(-|a|^{-(r+1)},|a|^{-(r+1)}\right) \subseteq(-\epsilon, \epsilon)$ and

$$
\begin{aligned}
\mathbb{E}\left(T_{(-\epsilon, \epsilon)}\right) & \leq \mathbb{E}\left(T_{\left(-|a|^{-(r+1)},|a|^{-(r+1)}\right)}\right) \leq \frac{A}{\pi_{0}\left(<I_{0}>\right)|a|^{-r}}+B \\
& \leq \frac{A}{\pi_{0}\left(<I_{0}>\right)|a|^{-\frac{\ln \epsilon^{-1}}{\ln |a|}+1}}+B=\frac{A}{\pi_{0}\left(<I_{0}>\right)|a|} \frac{1}{\epsilon}+B .
\end{aligned}
$$

Corollary 1 shows that $A$ and $B$ only depend on the Markov chain $P$ but not on $\epsilon$. From this, Theorem 1 immediately follows.

\section{REFERENCES}

[1] J. BAillieul, Feedback designs in information-based control, in: Proc. of the Workshop on Stochastic Theory and Control, Kansas, 2001, pp. 35-57, Springer-Verlag. 
[2] R. W. Brockett And D. Liberzon, Quantized feedback stabilization of linear systems, IEEE Trans. Automatic Control, AC-45(2000), pp. 1279-1289.

[3] J. BuzzI, Intrinsic ergodicity of affine maps in $[0,1]^{d}$, Monat. fur Mathematik, 124(1997), pp. 97-118.

[4] Pierre Collet, Some ergodic properties of maps of the interval, in: Dynamical systems (Temuco, 1991/1992), Vol. 52 of Travaux en Cours, pp. 55-91. Hermann, Paris, 1996.

[5] D. F. Delchamps, Stabilizing a linear system with quantized state feedback, IEEE Trans. Automat. Control, AC-35(1990), pp. 916-924.

[6] N. Elia And S. K MitTer, Stabilization of linear systems with limited information, IEEE Trans. Automat. Control, AC-46(2001), pp. 1384-1400.

[7] F. FAGNANi AND S. ZAMPIERI Stability analysis and synthesis for scalar linear systems with a quantized feedback, IEEE Trans. Automat. Control, AC-48 (2003), pp. 1560-1584.

[8] F. Fagnani And S. Zampieri A symbolic dynamics approach to performance analysis of quantized feedback systems: the scalar case, submitted.

[9] J. Kemeny, J. L. Snell, And A. W. Knapp, Denumerable Markov chains, Springer Verlag, New York, 1976.

[10] A. Lasota And J. A. Yorke, On the existence of invariant measures for piecewise monotonic transformations, Trans. of the Amer. Math. Soc., 186(1973), pp. 481-488.

[11] C. Liverani, Decay of correlations in piecewise expanding maps, Journal of Statistical Physics, 78(1995), pp. 1111-1129.

[12] G. N. NAIR AND R. J. Evans, Stabilization with data-rate-limited feedback: tightest attainable bounds, Systems and Control Letters, 41(2000), pp. 49-56.

[13] S. Tatikonda, Control under communication constraints, Ph.D. thesis, MIT, Cambridge, 2000.

[14] W. S. Wong And R. W. Brockett, Systems with finite communication bandwidth constraints II: Stabilization with limited information feedback, IEEE Trans. Automatic Control, AC44(1999), pp. 1049-1053. 